\title{
A MAGNETIC FIELD MODEL FOR WIGGLERS AND UNDULATORS *
}

\author{
D. Sagan, J. A. Crittenden, D. Rubin, Cornell University, Ithaca, NY, USA \\ E. Forest, 1-1 Oho, Tskuba, Ibaraki, Japan
}

\section{Abstract}

Recent interest in applications of wiggler magnets in storage rings has motivated efforts to incorporate their effects in calculations of beam dynamics. This paper presents an analytic model of wiggler fields that can be used with symplectic integration to evaluate such effects. Coefficients needed by the model are generated by fitting to the results of a finite-element field calculation. The model has been used successfully in the CESR-c project, which imposes tolerances of a few parts in $10^{4}$ on the modeling of 2-Tesla superconducting wigglers. In contrast to models based on Fourier transforms, the model presented here uses a relatively small number of terms, leading to correspondingly fast integration times. Fringe fields are included and no assumption about the periodicity of the field is made.

\section{INTRODUCTION}

A prerequisite for the study of particle dynamics is the ability to calculate transfer maps for each element in a storage ring. This is difficult for wigglers (wiggler here can mean either wiggler or undulator) since analytic formulas do not exist except in the most simplified cases. Wigglers can have strong nonlinear components[1,2], which can be a major limitation on the dynamic aperture, and impose stringent conditions on any analytic approximations.

Symplectic integration is an excellent technique for doing tracking and for constructing transfer maps[3]. In order to do symplectic integration through a wiggler, however, one needs to know the field as well as the gradient and higher derivatives. This generally precludes simply using data from a measurement or a calculation since the discrete nature of the data will make the higher derivatives inaccurate. What is needed is a model functional form that fits the data and can be easily and quickly differentiated. Such a model is presented below. This model has the advantage that end fields are easily incorporated into the model.

\section{FIELD MODEL}

Planar symmetry is assumed and the model functional form for the magnetic field of a wiggler $\mathbf{B}_{\mathrm{fit}}(x, y, s)$ is written as a sum of $N$ terms

$$
\mathbf{B}_{\mathrm{fit}}=\sum_{n=1}^{N} \mathbf{B}_{n}\left(x, y, s ; C_{n}, k_{x n}, k_{s n}, \phi_{s n}, f_{n}\right) .
$$

Each term $\mathbf{B}_{n}$ is parameterized by 5 quantities $C, k_{x}, k_{s}$, $\phi_{s}$, and $f$. The index $f_{n}=\{1,2$, or 3$\}$ is used to designate

\footnotetext{
* Work supported by the National Science Foundation
}

which of 3 forms a $\mathbf{B}_{n}$ term can take. The first form is

$$
\begin{aligned}
B_{x} & =-C \frac{k_{x}}{k_{y}} \sin \left(k_{x} x\right) \sinh \left(k_{y} y\right) \cos \left(k_{s} s+\phi_{s}\right) \\
B_{y} & =C \cos \left(k_{x} x\right) \cosh \left(k_{y} y\right) \cos \left(k_{s} s+\phi_{s}\right) \\
B_{s} & =-C \frac{k_{s}}{k_{y}} \cos \left(k_{x} x\right) \sinh \left(k_{y} y\right) \sin \left(k_{s} s+\phi_{s}\right) \\
& \text { with } k_{y}^{2}=k_{x}^{2}+k_{s}^{2} .
\end{aligned}
$$

The second form is

$$
\begin{aligned}
B_{x} & =C \frac{k_{x}}{k_{y}} \sinh \left(k_{x} x\right) \sinh \left(k_{y} y\right) \cos \left(k_{s} s+\phi_{s}\right) \\
B_{y} & =C \cosh \left(k_{x} x\right) \cosh \left(k_{y} y\right) \cos \left(k_{s} s+\phi_{s}\right) \\
B_{s} & =-C \frac{k_{s}}{k_{y}} \cosh \left(k_{x} x\right) \sinh \left(k_{y} y\right) \sin \left(k_{s} s+\phi_{s}\right) \\
& \text { with } k_{y}^{2}=k_{s}^{2}-k_{x}^{2},
\end{aligned}
$$

and the third form is

$$
\begin{aligned}
B_{x} & =C \frac{k_{x}}{k_{y}} \sinh \left(k_{x} x\right) \sin \left(k_{y} y\right) \cos \left(k_{s} s+\phi_{s}\right) \\
B_{y} & =C \cosh \left(k_{x} x\right) \cos \left(k_{y} y\right) \cos \left(k_{s} s+\phi_{s}\right) \\
B_{s} & =-C \frac{k_{s}}{k_{y}} \cosh \left(k_{x} x\right) \sin \left(k_{y} y\right) \sin \left(k_{s} s+\phi_{s}\right) \\
& \text { with } k_{y}^{2}=k_{x}^{2}-k_{s}^{2} .
\end{aligned}
$$

$k_{y}$ is considered to be a function of $k_{x}$ and $k_{s}$ and the relationship between them ensures that Maxwell's equations are satisfied.

Given a calculation or measurement of the field at a set of points $\mathbf{B}_{\text {data }}$, the problem is to find a set of $N$ terms such that $\mathbf{B}_{\text {fit }}$ and $\mathbf{B}_{\text {data }}$ agree to some given precision set by how accurately one needs to be able to track through a wiggler. This is a standard problem in nonlinear optimization. The solution is to minimize a merit function $M$

$$
M=\sum_{\text {data pts }}\left|\mathbf{B}_{\mathrm{fit}}-\mathbf{B}_{\mathrm{data}}\right|^{2}+w_{c} \sum_{n=1}^{N}\left|C_{n}\right| .
$$

The second term in $M$ is to help preclude solutions with degenerate terms that tend to cancel one another. The weight $w_{c}$ should be set just large enough to prevent this but not so large as to unduly distort the fit.

The minimization of $M$ can be done by any number of well known algorithms[4, 5]. The fitting process is simplified since the three forms can be combined into one continuous function via

$$
\mathbf{B}_{n}= \begin{cases}\text { Form \#1 } & 0<k_{x n} \\ \text { Form \#2 } & -\left|k_{s n}\right| \leq k_{x n} \leq 0 \\ \text { Form \#3 } & k_{x n}<-\left|k_{s n}\right|\end{cases}
$$




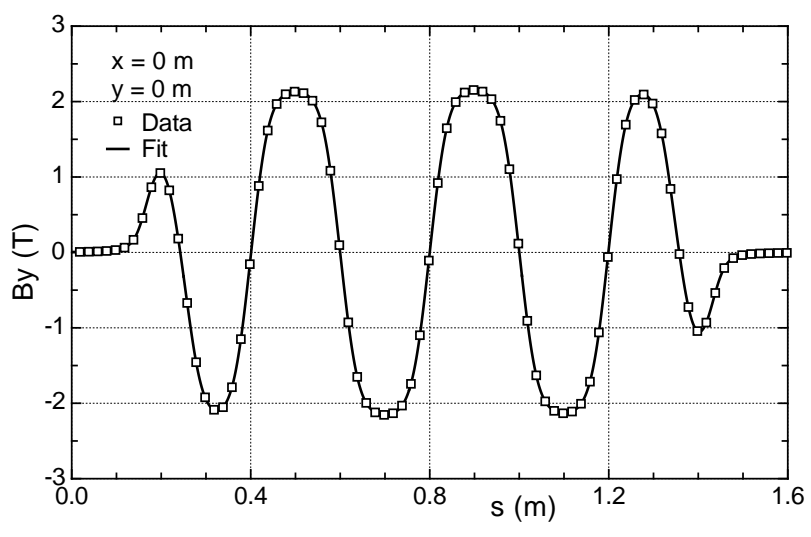

Figure 1: $B_{y}$ as a function of $s$ at $x=y=0$. The data points are from a finite element modeling program. The curve is calculated from an 82 term fit.

Once $B_{\text {fit }}$ is calculated, symplectic integration is performed using the Hamiltonian $H\left(x, p_{x}, y, p_{y}, z, \delta ; s\right)$ in the paraxial approximation

$$
H=\frac{\left(p_{x}-a_{x}\right)^{2}}{2(1+\delta)}+\frac{\left(p_{y}-a_{y}\right)^{2}}{2(1+\delta)}-a_{s},
$$

where $p_{x, y}=P_{x, y} / P_{0}$ is the normalized transverse momentum, $\delta=\Delta E / P_{0} c$ is the relative energy deviation, $z$ is the longitudinal position relative to the reference particle, and $\mathbf{a}(x, y, s)=q \mathbf{A} / P_{0} c$ is the normalized vector potential. To save on computational speed, the gauge with $a_{x}=0$ is used in the calculations. With this choice of gauge, formulas for $\mathbf{a}(x, y, s)$ from Eqs. (2), (3), and (4) are readily derived.

The symplectic integration procedure is given by $\mathrm{Wu}$ et al.[6] with the modification that Wu's prescription uses the $a_{s}=0$ gauge. This procedure has been integrated into the PTC (Polymorphic Tracking Code) subroutine library of Étienne Forest[7] which in turn has been integrated into the Cornell BMAD particle simulation software library[8].

A $2^{\text {nd }}$ order symplectic integrator[3] is used for the calculations. $4^{\text {th }}$ order and $6^{\text {th }}$ order integrators were also tried but, it was found, that after adjusting the number of integration steps to achieve a given accuracy, the $2^{\text {nd }}$ order integrator was fastest. This is not surprising given the large higher-order nonlinearities inherent in a wiggler field.

\section{CESR-C WIGGLER}

The wiggler magnets being installed in the Cornell CESR-c storage ring[9] have been modeled using the above procedure. Using the finite element modeling program OPERA-3D, a table of field versus position was generated. The validity of the field calculations was experimentally confirmed by measurements of tune as a function of beam position in a wiggler[10]. The spacing between points in the table was $4 \mathrm{~mm}$ horizontally, $2 \mathrm{~mm}$ vertically, and $2 \mathrm{~mm}$ longitudinally. The extent of the table was $\pm 48 \mathrm{~mm}$ horizontally, $\pm 26 \mathrm{~mm}$ vertically and $1.6 \mathrm{~m}$ longitudinally. Table data and fit curves of $B_{y}$ as a function of $s$

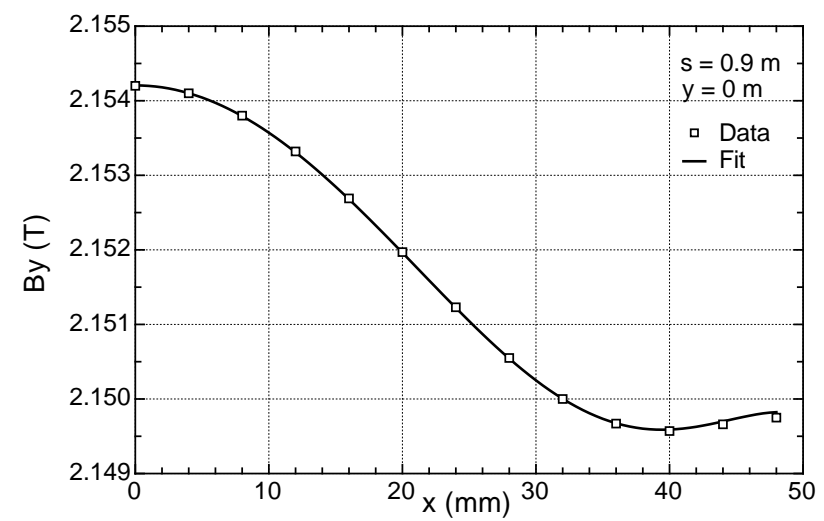

Figure 2: $B_{y}$ as a function of $x$ at $y=0$ and $s=0.9 \mathrm{~m}$. The data points are from a finite element modeling program. The curve is calculated from an 82 term fit.

and $x$ for the CESR-c 8-pole wiggler are shown in Figs. 1 and 2. 82 terms were used for the fit. The peak field is about $2 \mathrm{~T}$ and the RMS of the difference $\left|\mathbf{B}_{\text {data }}-\mathbf{B}_{\text {fit }}\right|$ was $9 \mathrm{G}$ which gives an RMS to peak field ratio of $0.05 \%$. The gradual roll-off of $B_{y}$ as a function of $x$ shows the advantage of allowing the $k_{x n}$ to vary continuously instead of using a Fourier series. With a Fourier series the $k_{x n}$ would be quantized which would necessitate the use of more terms in the fit and slow any symplectic integration.

Figs. 3 and 4 show tracking simulation results for the the CESR-c 8-pole wiggler. Fig. 3 shows $p_{x}$ at the end of the wiggler as a function of $x$ at the start with a starting condition of $y=20 \mathrm{~mm}$. Fig. 4 a shows $p_{y}$ at the end as a function of $y$ at the start with $x_{\text {start }}$ set at $30 \mathrm{~mm}$. The solid lines in Figs. 3 and 4a are the results of using a RungeKutta (RK) integrator with adaptive step size control[4] and with the field values obtained from interpolating the table from OPERA-3D. The dashed lines are from symplectic integration (SI) using the fitted field and 250 integration steps. The dash-dotted lines are from a $7^{\text {th }}$ order Taylor map (TM) which is generated using symplectic integration with 250 integration steps.

RK tracking, since it is derived directly from the equations of motion and the magnetic field table, is the gold standard with which to compare other tracking results. Fig. $4 \mathrm{~b}$ shows the difference between the SI and RK tracking as well as the difference between the TM and RK tracking. Additionally, for comparison, a line is shown whose slope represents a tune shift of $\Delta Q=0.001$ assuming a $\beta$ of $10 \mathrm{~m}$. The SI tracking agrees well with the RK, better than $4 \mu \mathrm{rad}$ in Fig. 3 and $8 \mu \mathrm{rad}$ in Fig. 4. Slope differences of the curves are also small, representing tune shifts of less than 0.001 (at $\beta=10 \mathrm{~m}$ ) everywhere in the figures. The advantage of the SI tracking is that it preserves the Poincare invariants, such as phase space density, while the RK does not. This is an important consideration in long term tracking where RK can give unphysical results.

The TM also show excellent agreement with the RK tracking except in Fig. 3 when the magnitude of $x$ is larger than $30 \mathrm{~mm}$ or so. In the domain where the TM agrees 


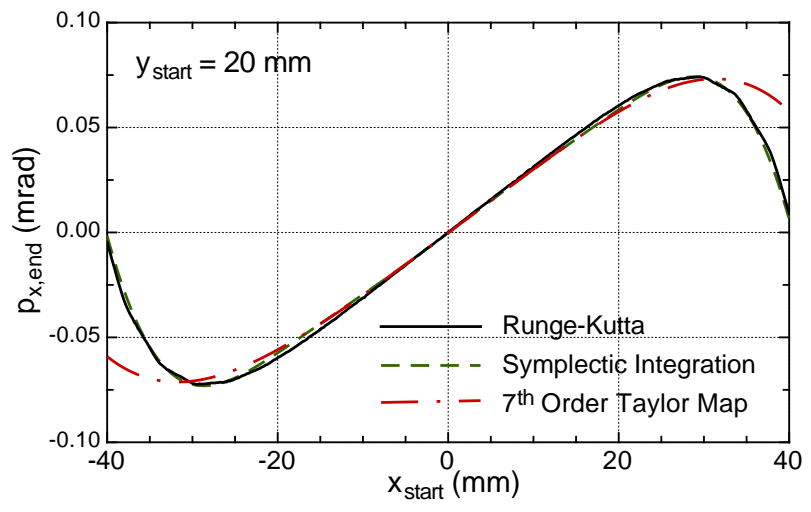

Figure 3: $p_{x}$ at the end of the wiggler as a function of $x$ at the start using three different tracking methods. At the start $p_{x}=p_{y}=\delta=0$ and $y=20 \mathrm{~mm}$.

with the RK, the TM can be used for such purposes as lattice design and other analyses that are not sensitive to nonsymplectic errors. The advantage of the TM is that it is fast. In the present instance the TM was over a factor of 30 faster than the other two methods. (This does not include the time to calculate the TM to begin with, but that only has to be done once). To overcome the non-symplecticity of the TM it can be partially inverted to form a symplectic generating function[3]. Preliminary investigations comparing the long term tracking results from SI and from a generating function show good agreement.

\section{CONCLUSION}

The wiggler model presented here is useful because it can accurately model a wiggler including end fields. This leads to efficient symplectic mapping which is needed in long term tracking, and avoids the non-physical violation of conserved quantities inherent when tracking is dependent upon interpolation of a field table. For applications where symplecticity is not a concern, a Taylor map, generated using the fit with symplectic integration can greatly reduce computation time.

For long periodic wigglers, the number of terms needed to fit the field may become large. In this case, a simple solution would be to divide the wiggler into 3 sections: the periodic center section and two end sections. Each section can be fitted separately. Since the center section is periodic, the number of terms needed to fit it is independent of its length. For the end sections it might be possible to cut down on the number of fit terms by making use of three additional forms that have an exponential $s$-dependence. These forms can be derived from Eqs. (2), (3), and (4) using the substitution $k_{s} \rightarrow-i k_{s}$.

If pole misalignments need to be simulated, then planar symmetry cannot be assumed. In this case, Eqs. (2), (3), and (4) can be modified, at some small increase in complexity, by using $k_{x} x+\phi_{x}$ in place of $k_{x} x$, and $k_{y} y+\phi_{y}$ in place of $k_{y} y$. With this, any arbitrary magnetic field profile can be modeled.

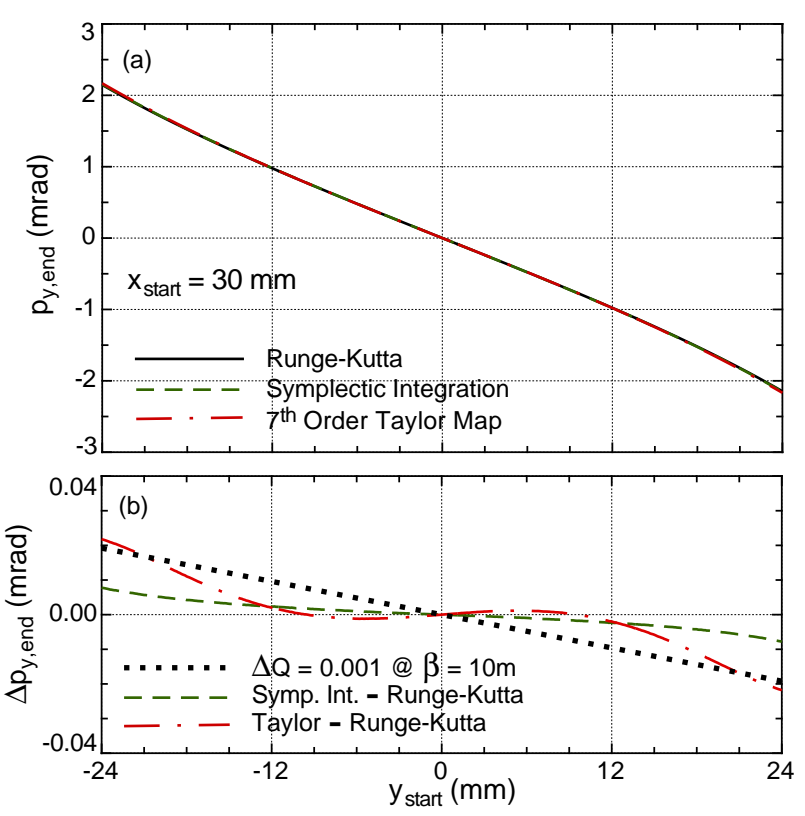

Figure 4: a) $p_{y}$ at the end of the wiggler as a function of $y$ at the start using three different tracking methods. At the start $p_{x}=p_{y}=\delta=0$ and $x=30 \mathrm{~mm}$. b) Difference between RK tracking and the two other methods. Also shown is a line that represents a tune shift of $\Delta Q=0.001$.

\section{REFERENCES}

[1] P. Kuske, R. Görgen, and J. Kuszynski, "Investigation of Non-Linear Beam Dynamics with Apple II-Type Undulators at Bessy II,” Proc. 2001 Part. Acc. Conf., pg. 1656 (2001).

[2] C. Milardi, D. Alesini, G. Benedetti, et al., "Effects of Nonlinear Terms in the Wiggler Magnets at DA $\Phi$ NE,” Proc. 2001 Part. Acc. Conf., pg. 1720 (2001).

[3] E. Forest, Beam Dynamics: A New Attitude and Framework, Harwood Academic Publishers, Amsterdam (1998).

[4] W. Press, B. Flannery, S. Teukolsky, and W. Wetterling, $\mathrm{Nu}$ merical Recipes in Fortran, the Art of Scientific Computing, Second Edition, Cambridge University Press, New York, 1992.

[5] F. James, MINUIT, Function Minimization and Error Analysis, CERN program library writeup D506

[6] Y. Wu, E. Forest, D. S. Robin, H. Nishimura, A. Wolski, and V. N. Litvinenko, "Symplectic Models for General Insertion Devices,” Proc. 2001 Part. Acc. Conf., pg. 398 (2001).

[7] See: <http://bc1.lbl.gov/CBP_pages/educational/

TPSA_DA/Introduction.html>.

[8] D. Sagan, and D. Rubin, “CESR Lattice Design,” Proc. 2001 Part. Acc. Conf., pg. 3517 (2001)

[9] J.A. Crittenden, A. Mikhailichenko, and A. Temnykh, "Design Considerations for the CESR-c Wiggler Magnets," these proceedings.

[10] A. Temnykh, J. A. Crittenden, D. Rice and D. Rubin, "Beam-based Characterization of a New 7-Pole Superconducting Wiggler at CESR," these proceedings. 